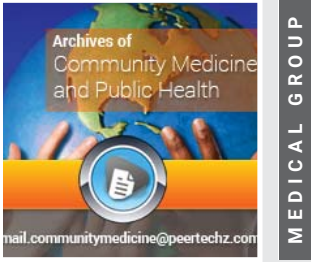

\title{
Outbreak of a new coronavirus (SARS-CoV-2) infection and pollen exposure
}

Received: 15 January, 2021

Accepted: 20 January, 2021

Published: 21 January, 2021

*Corresponding authors: Akira Awaya, PhD, Dermatology \& Epidemiology Research Institute (DERI), 4978 Totsuka-cho, Totsuka-ku, Yokohama, Kanagawa 244-0003, Japan, E-mail: awaya@home.email.ne.jp

https://www.peertechz.com

\section{Check for updates}

\begin{abstract}
Akira Awaya ${ }^{1,2 \star}$ and Yoshiyuki Kuroiwa ${ }^{3-5}$
'Dermatology \& Epidemiology Research Institute (DERI), 4978 Totsuka-cho, Totsuka-ku, Yokohama, Kanagawa 244-0003, Japan

${ }^{2}$ Graduate School of Nano bioscience, Yokohama City University, Yokohama, Kanagawa 236-0027, Japan

${ }^{3}$ Department of Neurology, University Hospital Mizonokuchi, Teikyo University School of Medicine, 5-1-1, Futago, Takatsu-ku, Kawasaki, Kanagawa 213-8507, Japan

${ }^{4}$ Department of Medical Office, Ministry of Finance, 3-1-1, Kasumigaseki, Chiyoda-ku, Tokyo 100-8940, Japan

${ }^{5}$ Department of Neurology, Yokohama City University Graduate School of Medicine, 3-9, Fukuura, Kanazawa-ku, Yokohama, Kanagawa 236-0004, Japan
\end{abstract}

\begin{abstract}
The inference that this paper points out is that the stimulating environmental factor behind why the SARS-CoV-2 epidemic occurred in Wuhan is probably pollen from the plant kingdom. We started in 2003 to point out the epidemiological fact that Kawasaki disease is probably a Pollen-Induced Disease (PID) triggered by pollen exposure. Subsequently, we also examined the correlation analysis between the cyclical changes in pollen count and the changes in the number of new patients with respect to the incidence of 40 designated intractable diseases and 24 cancers and malignancies over a 40 -year period, and were able to show a significant association. Since the 1990s, it has been reported that the number of pollen scattered in Wuhan, China is far higher than the number of pollen scattered in other parts of Japan, and is by far the highest in China. Before the COVID-19 epidemic began, it is conceivable that some of the allergic prone (allergic constitution) residents of Wuhan City, who have been exposed to large amounts of pollen in the spring and small amounts of precursor pollen in the fall each year, fell into a compromised immune state in November. Through a mechanism similar to the initial process of carcinogenesis triggered by pollen exposure, patients infected with the old coronavirus, which is a foreign substance, are thought to have developed a mechanism whereby the old coronavirus mutates (becomes highly toxic) in the host body in the course of their biological response to the virus. This supposed host response process (trick) allows the virus to expand its mutation at the genetic level, which is beyond the control of the host human, during the replication process. As a result, the virus will cause a situation (condition) that will lead to an infectious disease pandemic that can be transmitted from person to person who have not yet developed resistance. It is implied that within the host, a suicidal pathological process, costly to humanity, may be occurring in a process similar to that of individual carcinogenesis.
\end{abstract}

Text

Inference on the outbreak process of the SARS-CoV-1 (Severe acute respiratory syndrome coronavirus1) epidemic first discovered in Foshan, Guangdong Province, China in November 2002, followed by the SARS-CoV-2 epidemic that began in Wuhan, Hubei Province, China in November 2019.

Both the SARS-CoV-1 and SARS-CoV-2 epidemics in China first broke out in November, the period between autumn and the beginning of winter [1]. In Japan, there are cases in November when people with allergic tendencies (allergic constitution) visit their family clinics with fever and are diagnosed as influenza virus patients. In Japan, the rapid increase in the number of Kawasaki disease cases usually begins in the fall and winter months, following the first small amounts of cedar pollen are dispersed from September to November, after large amounts of released cedar pollen from February to April [2-4]. However, in the November-December period of 2020, the number of Kawasaki disease patients has decreased dramatically [5]. Probably, the positive effect was due to the fact that pollen exposure was avoided by restricting even infants from going 
outside and wearing masks and goggles to prevent COVID-19 infection.

COVID-19, which began its pandemic in Wuhan in December 2019, quickly spread around the world, creating a pandemic that has now affected 84 million people, left patients suffering, clinicians, nurses and other healthcare workers exhausted. The death toll is reported to have reached 1.83 million. Patients present with a wide range of symptoms, mainly pneumonia and blood clots, and are treated with existing antiviral and anti-inflammatory drugs, and severe patients are treated with Extracorporeal Membrane assisted ventilation (ECMO). Virus researchers have been working hard to develop various vaccines by immediately analyzing the genome. Clinical trials have already been conducted, products have been developed, and the vaccine has been approved as a pharmaceutical product in many countries. In fact, vaccinations have been initiated in many countries. Epidemiologists have been recommending public health measures to contain the pandemic.

\section{Why was SARS-CoV-2 born in Wuhan in 2019?}

In the background, the author noted that data from the late 1980 s to around 2000 showed that the number of pollen dispersed in Wuhan was by far the highest among the major cities in China [6-8]. The author compared the changes in the number of pollen counts observed at the National Hospital Organization Sagamihara Hospital [2-4,9] and Tokyo Metropolitan Government [4] from the 1970s to the 2010s with the changes in the number of cases of Kawasaki disease, 40 designated intractable diseases $[10,11]$, and 24 types of cancer and malignant tumors $[12,13]$. We have found epidemiological facts that pollen exposure, which most Japanese people are inevitably exposed to for more or less eight months a year immediately after birth, is strongly associated with the development of these incurable diseases. This fact reminded me that pollen exposure seems to be a trigger for the onset of many diseases, including many incurable diseases. The authors proposed the existence of a disease named "Pollen-Induced Diseases (PID)" [2], namely Pollen Disease (PID) [14]. Thinking that PID may be one of the few damages that creatures of the animal kingdom, including human beings, suffer from the plant kingdom, which contributes so much to the animal kingdom, the author cannot help but feel the mysterious providence of nature and life phenomena.

As many as 40 designated intractable diseases are equally affected by pollen exposure, to a greater or lesser extent, and this has been verified in the process of making graphs for Kawasaki disease (Figure 1), Takayasu disease, vasculitis syndromes [10], collagen diseases [10], Parkinson's disease, multiple sclerosis, ulcerative colitis [11], interstitial pneumonia [11], etc. In the process, the author was convinced that, given the commonality of life phenomena and disease pathology, cancer is also likely to be caused by pollen exposure (Figure 2) $[12,13]$. In addition, I was hoping to analyze pollen count data from mainland China and the Mediterranean region, because the major viral mutations that occur in host animals can be thought of as a phenomenon that responds to major changes in the external environment surrounding the animal kingdom.
Reasoning that the mechanism by which the old coronavirus mutates in the host body is similar to the initial process of carcinogenesis triggered by pollen exposure.

The author believes that the elucidation of the initial biological reaction mechanism (pathogenesis) of viral diseases is a priority, based on the knowledge accumulated by experts, regarding how the onset of disease occurs in individuals in an isolated manner in the early stages before the spread of infection or the start of an epidemic. In conclusion, viral infectious diseases among microorganisms, as well as the onset of intractable diseases and carcinogenesis, may be affected by the accumulation of pollen exposure over time, starting immediately after birth in the host animal kingdom. I would like to understand the outbreak of a new coronavirus as the progression of a process in which a certain annual oncogenicinducing mechanism in the host causes a major mutation of a foreign virus.

For example, during the summer, children may develop mild summer colds such as hand, foot, and mouth disease and erythema infectiosum, as well as a more severe form of aseptic meningitis [5]. This phenomenon can be regarded as a result of the Th2 tilt of the Th1/Th2 balance inducing weak viral susceptibility after pollen exposure. I have also commented that the onset of drug-induced diseases such as Stevens-Johnson syndrome, toxic epidermal necrolysis, and drug-induced hypersensitivity syndrome, as well as the seasonal onset of aseptic meningitis, may be due to increased drug sensitivity in the body after pollen exposure [14,15].

It is believed that the chickenpox (varicella) virus (varicellazoster virus), which was contracted in childhood, lurks in the area called the ganglia near the spinal cord even after the chickenpox has healed. It is reactivated when the body's immunity is lowered due to fatigue or stress, it passes through the peripheral nerves to form shingles on the skin. The human immunodeficiency virus is believed to be a virus that infects and destroys human immune cells, eventually causing acquired immunodeficiency syndrome. Kaposi's sarcoma-associated herpesviruses have been known to infect cells, become latently infectious as episomes, and exert their virulence in response to immunodeficiency in the host [16].

As shown in this example, the onset of viral disease is thought to be triggered by the formation of a compromised immune state (opportunistic readiness) in the host patient. What triggers this susceptible state? The authors assume that the first case in Foshan City, Guangdong Province (adjacent to Guangzhou City, the provincial capital) in 2002, and the first case in Wuhan City, Hebei Province in 2019, were both caused by allergy-prone (allergic) residents who were exposed to large amounts of pollen and became easily infected by a coronavirus. When infected by a coronavirus, or when the virus is activated from a latent or dormant state, the host develops various cellular responses that place an unprecedented burden on the old coronavirus, resulting in a new coronavirus. In this way, the process of mutation into SARS-CoV-1 and SARS-CoV-2 is considered to have progressed. 


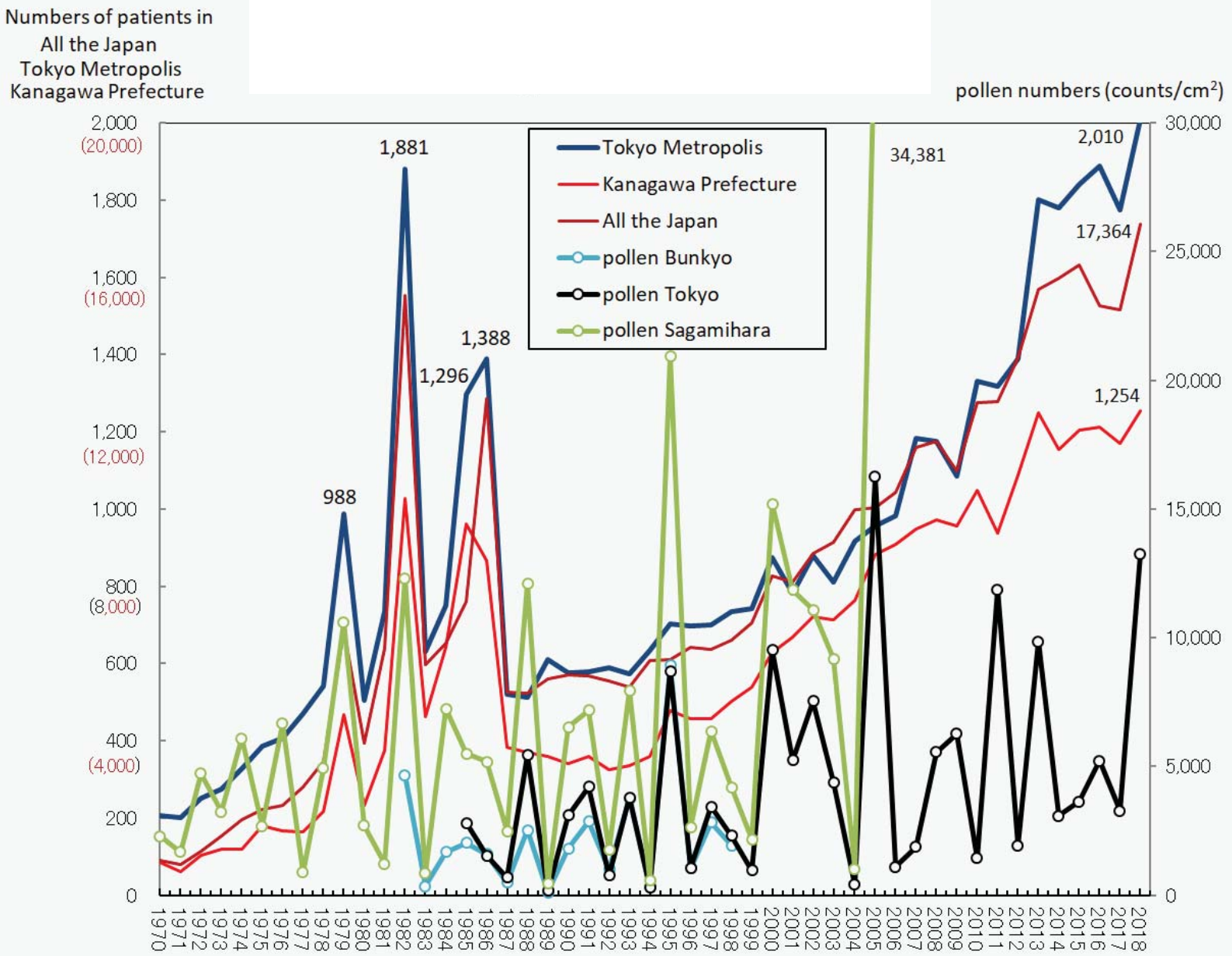

Figure 1: Annual numbers of Kawasaki disease patients in Tokyo Metropolis, Kanagawa Prefecture and all the Japan, and scattering pollen from 1970 to 2018.

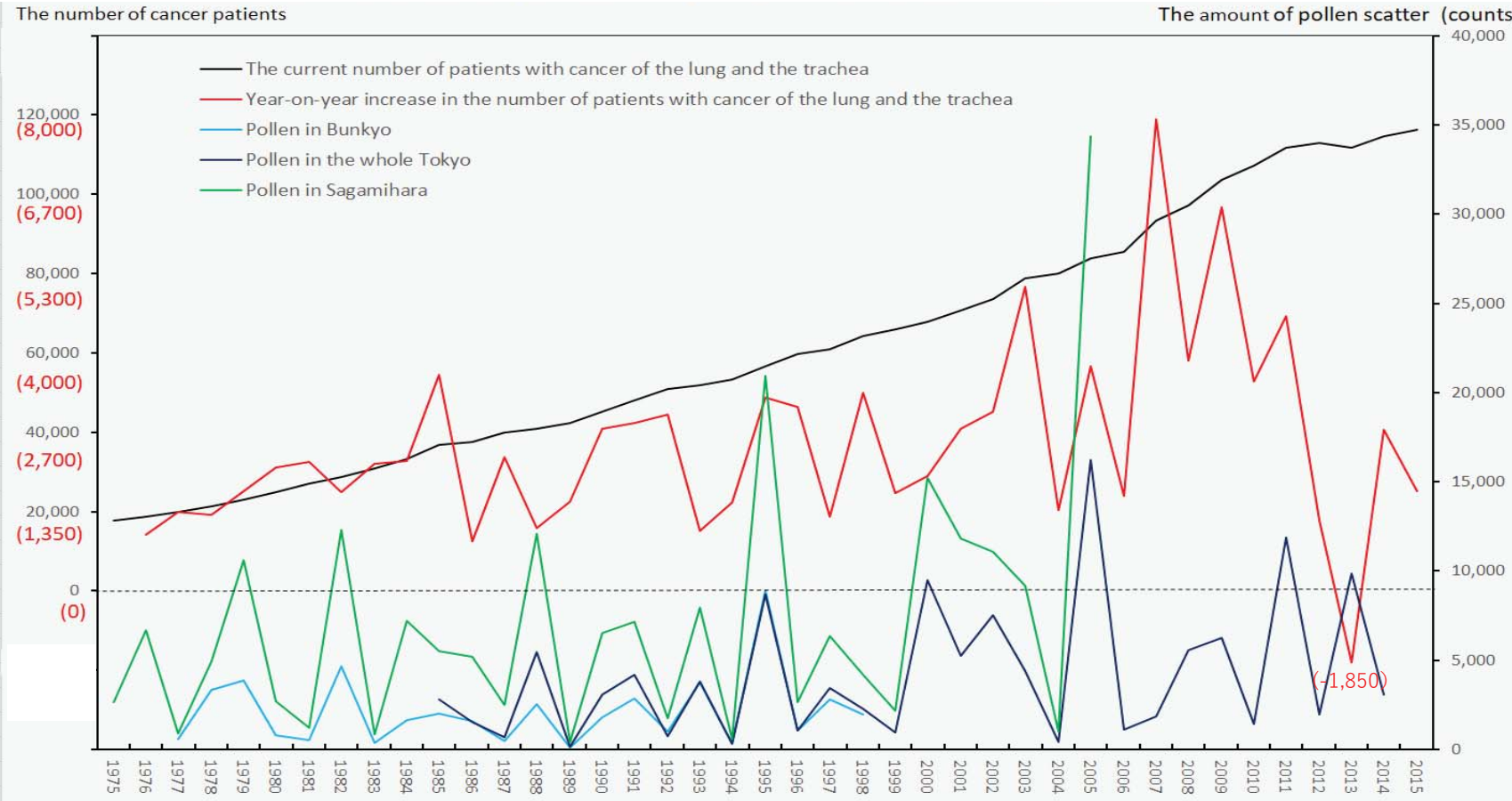

Figure 2: Total number of Japanese males and females with cancer of the lung and the trachea at the year diagnosed its year-on-year increase and the amount of air borne pollen in 3 areas during the period from 1975 to 2015.

Citation: Awaya A, Kuroiwa Y (2021) Outbreak of a new coronavirus (SARS-CoV-2) infection and pollen exposure. Arch Community Med Public Health 7(1): 012-016. DOI: https://dx.doi.org/10.17352/2455-5479.000126 
There is a possibility that the coronavirus was transmitted to the easily infected residents of Wuhan through contact with bats after the human- and animal-infectious virus and livestock-endemic virus repeatedly mutated in the bodies of bats exposed to large amounts of pollen.

The following is a discussion of the mechanism by which a virus-induced disease can develop in a small number of people due to an unconventional exogenous viral infection in an isolated state before it becomes a pandemic in which everyone is infected. In other words, as with the onset of intractable diseases, when a person who has been exposed to pollen from birth exceeds the assumed threshold level of exposure in each year, and then becomes infected with a virus in the easily infected state of autumn and winter, the disease develops while he is causing a new counterattack, elimination, and assimilation of foreign substances through intracellular interactions. I would like to know the annual dynamics of pollen counts and the pollen exposure of people exposed to large amounts of pollen over the past 30-40 years in various parts of southern China, including 2019 when SARS-CoV-2 occurred in Wuhan.

In Japan, the measurement of pollen count on the rooftop of the current Sagamihara Hospital of the National Hospital Organization was started in 1965 [9]. In Tokyo, measurements were started in early spring in 1985 [4]. Since then, the number of measurement points in Tokyo has increased to 12 and the measurement period has been extended. I would like to know the current status of pollen observation in China and the status of information disclosure of pollen count data as soon as possible.

As described at the beginning of this report, Kawasaki disease has a biphasic onset pattern with a rapid increase in the number of patients in spring and summer, slightly later than the time of year when large amounts of cedar pollen are scattered, and in autumn and winter from November. However, since 1987, the number of patients with Kawasaki disease has been decreasing rapidly in around February when the influenza epidemic is at its peak [4].

\section{Influenza pandemic so far in Japan and COVID-19 pan- demic}

In 2008-2009, the number of seasonal influenza cases in Tokyo was the highest ever recorded [4]. The persistence of a Th2 tilt in the Th1/Th2 balance in allergic (allergic constitution) individuals affected by the largest ever pollen dispersal in Japan in 2005, and the booster effect of the large amount of pollen dispersed in 2008-2009 on the persistence of this immune state, are thought to have influenced the sustained increase in the number of Kawasaki disease patients since 2008 (Figure 1) and the record number of seasonal influenza cases in 20082009 [4]. The fact that there may be a link between the increase in pollen count and the influenza epidemic will eventually be clarified through research by experts.

In the spring of 2009, a new type of influenza (swine) arrived in Japan from other countries, including those in the southern hemisphere, and a pandemic broke out in Japan in late 2009. The largest seasonal influenza pandemic in history in 2008-2009 caused a sharp decline in the number of cases of Kawasaki disease, as predicted in advance. In the 4th report of Kawasaki disease [4], the author reported that the onset of Kawasaki disease, which is considered to be a pollen-induced delayed hypersensitivity reaction disease, was suppressed during the influenza epidemic.

Seasonal influenza in Japan in 2019-2020 was found to have died down after 11 weeks, with only a few outbreaks in December-February, probably due to the COVID-19 pandemic. The authors reported the phenomenon of no decrease in the number of Kawasaki disease patients till 26 weeks, at the 40th Annual Meeting of the Japanese Society for Kawasaki Disease in the fall of 2020, perhaps because there was little seasonal influenza epidemic, unlike previous years. The author believed in 2020 spring that the COVID-19 epidemic has suppressed the transmission of influenza viruses to humans. The number of Kawasaki disease cases after 27 weeks in 2020 was clearly lower than that in 2019, despite the small amount of cedar pollen prevailing from September to November, probably due to the wearing of masks by even infants for COVID-19 control and the reduction of going out.

Finally, pathological, molecular biological, immunological, genetic, and virological approaches to carcinogenesis research are expected to have led to the assumption that the coronavirus mutation process is presumed to have occurred by a mechanism that is common (and identical) to the carcinogenic process induced by pollen exposure. The authors' hypothesis is that if we can ascertain the annual dynamics of pollen counts in Foshan City, Guangdong Province and Wuhan City, Hubei Province and surrounding areas in China since the 1990s, we will be able to support the above hypothesis as well as the epidemiological fact that an association between pollen counts and the number of patients with intractable diseases was recognized by comparing annual changes in Japan.

In previous report [17], the authors focused on the effects of air temperature, humidity, and weather conditions on virus infections. It is an analytical report on the social situation in the pandemic and the transmission epidemic after the virus infections. As for previous report [18] related to "Pollutants and their relationship to COVID-19", there are a few references to mechanisms such as TLRs. The report also mentions that PM2.5 may be a carrier of SARS-CoV-2, but it only mentions the effect of COVID-19 on the severity of the disease, lacking discussion of the role of the host or the dynamics of the host in tissues and cells in the early stages of a new coronavirus outbreak. Air pollutants such as PM2.5 and PM10 are considered to be the products of the combustion of coal and wild grasses, as well as many industrial waste-like harmful inorganic materials and refinery-related organic materials such as crude oil and gasoline. We believe that these pollutants and pollen from the airborne pollen of the living plant kingdom should not be discussed together. It may not be appropriate to lump pollen-related research on pollen exposure, which causes many diseases, together with research on pollutants and research methods. These are matters that should be distinguished and discussed in different dimensions and categories. The annual emissions of 
many industrial wastes do not fluctuate significantly, whereas pollen emissions fluctuate cyclically both during the year and between years. In conjunction with such periodical pollen exposures, the incidence of many diseases might also fluctuate periodically. Our perspective article is based on our experience in reporting on the relationship between pollen exposure and the onset of 65 specific diseases, many of which are intractable. It was postulated that the coronaviruses mutated into highly toxic or malignant viruses during the replication process in the compromised host, similar to carcinogenesis, in relation to pollen exposure. However, we have to wait for the logic and scientific results of rigorous biological, immunological, epidemiological, and clinical researches, to be convinced of the relationships between environmental pollen pollution and the spread of coronavirus pandemic.

We hope that the inconvenient (self-sacrificing) response process to coronaviruses by animal hosts triggered by pollen exposure will be investigated.

\section{Acknowledgement}

Akira Awaya cordially expresses gratitude to Mr. Koichi Iwata for valuable minute data processing, graphing design, and correlation analysis.

\section{References}

1. Wikipedia information.

2. Awaya A, Murayama K (2012) Positive correlation between Japanese ceda pollen numbers and the development of Kawasaki Disease. Open Allergy J 5 1-10. Link: https://bit.ly/3slzBcu

3. Awaya A, Nishimura C (2014) A combination of cross correlation and trend analyses reveals that Kawasaki disease is a pollen-induced delayed-type hyper-sensitivity disease. Int J Environ Res Public Health 11: 2628-2641. Link: https://bit.ly/3sCQHIO

4. Awaya A (2016) Suppressive influence of seasonal influenza epidemic on Kawasaki disease onset. Jpn J Clin Immunol (Nihon Rinsho Meneki Gakkai Kaishi) 39: 528-537. Link: https://bit.ly/3932NTL

5. Individual data according to the disease downloaded from weekly and monthly report, Tokyo Metropolitan Infectious Disease Surveillance Center (in Japanese).

6. Miyoshi A (2021) Distribution of survey sites of pollens in China. 3443 Communication: Public Health Information in Miyagi Prefecture by Akira Miyoshi's Home Page. Link: https://bit.ly/3iBGZBT

7. Yin M, Miyoshi A, Cheng L, Shirakawa T, Enomoto T, et al. (1999) Cedar Pollinosis in China An epidemiological and clinical study in south China Otologia Fukuoka 45: S 625-S 629. Link: https://bit.ly/3o3iP4q

8. Takahashi Y, Cheng L, Xu L, Zhang S, Yang Q, et al. (2003) Airborne ceda pollen and cypress pollen survey in Wuhan in China. Otologia Fukuoka 49: S108-S112. Link: https://bit.ly/2NqdrM7

9. Awaya A, Sahashi N (2004) The etiology of Kawasaki disease: Does intense release of pollen induce pollinosis in constitutionally allergic adults, while constitutionally allergic infants develop Kawasaki disease? Biomed Pharmacother 58: 136-140. Link: https://bit.ly/3p2IH2I
10. Awaya A (2018) Development of many so-called autoimmune diseases including various vasculitis syndromes may be commonly triggered by pollen exposure. Jacob J Aller Immuno 5: 26. Link: https://bit.ly/2MdJbDp

11. Awaya A, Kuroiwa $Y$ (2019) A Retrospective study on the relationship between annual airborne pollen levels during four Decades of 1975-2014 and annual occurrence of ulcerative colitis, Crohn's disease, primary biliary cirrhosis, fulminant hepatitis, severe acute pancreatitis, interstitial pneumonia, amyloidosis, based on the national registry database of specific intractable diseases in Japan. Arch Epid Pub Health 1. Link: https://bit.ly/3iBdLmD

12. Awaya A, Kuroiwa Y (2020) The Relationship between Annual Airborne Pollen Levels and Occurrence of All Cancers, and Lung, Stomach, Colorectal, Pancreatic and Breast Cancers: A Retrospective Study from the National Registry Database of Cancer Incidence in Japan, 1975-2015. Int J Environ Res Public Health 17: 3950. Link: https://bit.ly/360TEct

13. Awaya A, Kuroiwa Y (2020) 40 Specific Intractable Diseases and 24 Types of Cancer and Malignancies as Well as Kawasaki Disease May be Triggered by Pollen. Arch Environ Sci Environ Toxicol 3: 130. Link: https://bit.ly/3bVKRwb

14. Awaya A (2020) Proposal for construction of test and evaluation method in basic research and clinical study of medicines considering the effect of pollen exposure. Res. Drug Interact 44: 1-8.

15. Awaya A (2016) Pollen-Induced Diseases No. 8; Investigation on the seasonal changes of drug effectiveness. Link: https://bit.ly/35Z9Pa9

16. Home Page of Division of Virology. Department of Microbiology and Immunology, Osaka University Graduate School of Medicine. Link: https://bit.ly/3qlkCNR

17. Espejo W, Celis JE, Chiang G, Bahamonde P (2020) Environment and COVID-19: Pollutants, impacts, dissemination, management and recommendations for facing future epidemic threats. Sci Total Environ 747: 141314. Link: https://bit.ly/2M7tw8L

18. Copiello S, Grillenzoni C (2020) The spread of 2019-nCoV in China was primarily driven by population density. Comment on «Association between short-term exposure to air pollution and COVID-19 infection: Evidence from China» by Zhu et al. Sci Total Environ. 744: 141028. Link: https://bit.ly/2LUDRoJ

\section{Discover a bigger Impact and Visibility of your article publication with}

\section{Peertechz Publications}

\section{Highlights}

* Signatory publisher of ORCID

* Signatory Publisher of DORA (San Francisco Declaration on Research Assessment)

* Articles archived in worlds' renowned service providers such as Portico, CNKI, AGRIS, TDNet, Base (Bielefeld University Library), CrossRef, Scilit, J-Gate etc.

* Journals indexed in ICMJE, SHERPA/ROMEO, Google Scholar etc.

* OAI-PMH (Open Archives Initiative Protocol for Metadata Harvesting)

* Dedicated Editorial Board for every journal

* Accurate and rapid peer-review process

* Increased citations of published articles through promotions

* Reduced timeline for article publication

Submit your articles and experience a new surge in publication services (https://www.peertechz.com/submission).

Peertechz journals wishes everlasting success in your every endeavours.

Copyright: @ 2021 Awaya A, et al. This is an open-access article distributed under the terms of the Creative Commons Attribution License, which permits unrestricted use, distribution, and reproduction in any medium, provided the original author and source are credited. 